\title{
Expression of the genes for peroxisome proliferator- activated receptor- $\psi$, cyclooxygenase-2, and proinflammatory cytokines in granulosa cells from women with polycystic ovary syndrome
}

\author{
Joong Yeup Lee', Jin Cheol Tae', Chung Hyon Kim', Doyeong Hwang'1, Ki Chul Kim', Chang Suk Suh², Seok Hyun Kim² \\ 'Hamchoon Women's Clinic, Seoul; ${ }^{2}$ Department of Obstetrics and Gynecology, Seoul National University College of Medicine, Seoul, Korea
}

Objective: To identify differences in the expression of the genes for peroxisome proliferator-activated receptor (PPAR)- $\gamma$, cyclooxygenase (COX)2 , and the proinflammatory cytokines interleukin (IL)- 6 and tumor necrosis factor (TNF)- $a$ in granulosa cells (GCs) from polycystic ovary syndrome (PCOS) patients and controls undergoing controlled ovarian stimulation.

Methods: Nine patients with PCOS and six controls were enrolled in this study. On the day of oocyte retrieval, GCs were collected from pooled follicular fluid. Total mRNA was extracted from GCs. Reverse transcription was performed and gene expression levels were quantified by realtime quantitative polymerase chain reaction.

Results: There were no significant differences in age, body mass index, and total gonadotropin dose, except for the ratio of luteinizing hormone to follicle-stimulating hormone between the PCOS and control groups. PPAR- $\gamma$ and COX-2 mRNA was significantly downregulated in the GCs of PCOS women compared with controls ( $p=0.034$ and $p=0.018$, respectively), but the expression of IL- 6 and TNF-a mRNA did not show significant differences. No significant correlation was detected between the expression of these mRNA sequences and clinical characteristics, including the number of retrieved oocytes, oocyte maturity, cleavage, or the good embryo rate. Positive correlations were found among the PPAR- $\gamma$, COX-2, IL-6, and TNF-a mRNA levels.

Conclusion: Our data may provide novel clues regarding ovarian GC dysfunction in PCOS, and indirectly provide evidence that the effect of PPAR- $\gamma$ agonists in PCOS might result from alterations in the ovarian follicular environment. Further studies with a larger sample size are required to confirm these proposals.

Keywords: Cyclooxygenase 2; Granulosa cells; Interleukin-6; Peroxisome proliferator-activated receptor gamma; Polycystic ovary syndrome; Tumor necrosis factor-alpha

\section{Introduction}

Polycystic ovary syndrome (PCOS) is a common heterogeneous en-

Received: Mar 10, 2017 · Revised: May 20, 2017 · Accepted: Jun 19, 2017 Corresponding author: Seok Hyun Kim

Department of Obstetrics and Gynecology, Seoul National University College of Medicine, 103 Daehak-ro, Jongno-gu, Seoul 03080, Korea

Tel: +82-2-2072-3773 Fax:+82-2-762-3599 E-mail: seokhyun@snu.ac.kr

*This work was supported by a grant from the Seoul National University Hospital (SNUH) Research Fund (No. 04-2011-0200).

This is an Open Access article distributed under the terms of the Creative Commons Attribution Non-Commercial License (http://creativecommons.org/licenses/by-nc/4.0/) which permits unrestricted non-commercial use, distribution, and reproduction in any medium, provided the original work is properly cited. docrinopathy in women of reproductive age [1]. Folliculogenesis in ovaries affected by PCOS is often disrupted, leading to suboptimal oocyte competence for fertilization. The association between PCOS and oocyte quality is controversial. Although the preponderance of evidence suggests that the developmental potential of oocytes derived from women with PCOS is normal [2], antral follicles in women with PCOS are heterogeneous and include a proportion that have undergone premature arrest [3]. Arrested follicles have different properties, and the oocytes in such follicles are unlikely to have normal developmental potential. Successful fertilization and pregnancy are heavily dependent upon the inherent qualities of the oocytes, and thus reliant upon the fidelity of oocyte maturation [4]. Although the etiology of 
PCOS is still obscure, dysregulation of granulosa cells (GCs) may affect the ovarian follicular environment, which may be associated with the poor reproductive outcomes observed in PCOS patients [5].

Insulin resistance plays a key role in the metabolic and reproductive consequences of PCOS, and involves nuclear events [6]. Peroxisome proliferator-activated receptor (PPAR)- $\gamma$ is one of the main nuclear receptors that regulate insulin sensitivity and has been identified as playing a crucial role in reproduction, including the regulation of steroidogenesis in GCs $[7,8]$. Using a mouse mutant model, GC-specific deletion of PPAR- $\gamma$ resulted in marked impairment of ovulation owing to defective follicular rupture. In the ovary, PPAR- $\gamma$ is expressed most strongly in GCs [9]. Thiazolidinediones which are synthetic agonists of PPAR- $\gamma$, are applied to treat PCOS [10], also suggesting that PPAR- $\gamma$ plays an important role in the maintenance of normal ovarian function and that its level or activity may be lower in PCOS patients than in normal individuals [11].

PPAR- $\gamma$ has also been recognized as playing an important role in the immune response through its ability to inhibit the expression of inflammatory cytokines and to direct the differentiation of immune cells toward anti-inflammatory phenotypes [12]. Tumor necrosis factor (TNF)- $\alpha$ and interleukin (IL)- 6 have been shown to influence ovarian function, ovulation, fertilization, and implantation in women with PCOS [13]. Additionally, it is possible that the increased expression of PPAR $-\gamma$ may be indirectly related to the proinflammatory status mediated by prostaglandin E [14]. It has been reported that the PPAR system regulates the synthesis of prostaglandins by modulating cyclooxygenase (COX)-2, the limiting enzyme of this process [15]. COX2 knockout mice have impaired ovulation, indicating that these genes control ovulation and cumulus expansion [16].

Few studies on the expression of PPAR- $\gamma$, COX-2 and proinflammatory cytokines in GCs have been published. We explored the differences in expression of PPAR- $\gamma$, COX-2, TNF- $\alpha$, and IL- 6 mRNA in GCs from $P C O S$ patients and controls undergoing controlled ovarian stimulation.

\section{Methods}

\section{Study design}

This was a pilot study performed in a private in vitro fertilization clinic. Fifteen patients with infertility for whom intracytoplasmic sperm injection (ICSI) was indicated were enrolled in the study. Nine patients with PCOS were assigned to the case group. Six non-PCOS patients whose partners had male factor infertility (azoospermia, ICSI with donor sperm) were assigned to the control group. All women were carefully screened for PCOS according to the Rotterdam criteria, meaning that they met at least two of the following three criteria: (1) oligo-ovulation and/or anovulation, (2) clinical and/or biochemical signs of hyperandrogenism, and (3) polycystic ovaries [17].

All ICSI cycles included in this study were conducted according to a long mid-luteal gonadotropin-releasing hormone ( $\mathrm{GnRH})$ agonist protocol as previously described [18]. Briefly, pituitary down-regulation with a GnRH agonist (decapeptyl-depot; Ferring, Malmo, Sweden) was followed by controlled ovarian stimulation with human menopausal gonadotropin (hMG; 225-300 IU/day, Menopur, Ferring). The dose of hMG was adjusted every 3 to 4 days based on ovarian response, as determined by ultrasound scans. When adequate follicular growth was demonstrated (more than two follicles reached $18 \mathrm{~mm}$ in diameter), human chorionic gonadotropin (Ovidrel; Serono, Geneva, Switzerland) was administered subcutaneously to induce follicular maturation. After 35 to 36 hours, follicular aspiration was performed under the guidance of transvaginal ultrasound. The luteal phase was supplemented with progesterone in oil once daily, starting on the day of oocyte retrieval. A clinical pregnancy was defined as the presence of a gestational sac on ultrasonography. An ongoing pregnancy was a pregnancy that reached a viable gestational age of $\geq 12$ weeks.

All patients provided informed written consent, and this study was approved by the institutional review board of Hamchoon Women's Clinic (No. HCIRB-1106-01-002).

\section{Isolation of GCs}

GCs were collected on the day of oocyte retrieval. After the isolation of cumulus-oocyte complexes, the remaining follicular fluid (FF) was pooled. To remove red blood cells, GCs were harvested from the FF by using a discontinuous Percoll gradient (50\%, 90\% in Ham's F10 medium) and centrifugation at room temperature (3,000 rpm for 10 minutes). After removal of the supernatant, the pellets were resuspended in Ham's F-10 medium (Gibco BRL, CA, USA). The GCs were washed in fresh medium (Ham's F-10 medium) and stored at $-80^{\circ} \mathrm{C}$ until RNA analysis.

\section{RNA isolation and real-time reverse transcription polymerase chain reaction}

The total RNA of PPAR- $\gamma$, COX-2, IL-6, and TNF- $a$ was extracted from

Table 1. Sequences of primers used for real-time reverse transcription polymerase chain reaction

\begin{tabular}{lll}
\hline Gene & Forward primer $\left(5^{\prime}\right.$ to $3^{\prime}$ ) & \multicolumn{1}{c}{ Reverse primer $\left(5^{\prime}\right.$ to $3^{\prime}$ ) } \\
\hline COX-2 & CCGGACAGGATTCTATGGAG & AGGAGAGGTTAGAGAAGGCT \\
IL-6 & TCTCCACAAGCGCCTTCGGT & TTCACCAGGCAAGTCTCC \\
TNF- $a$ & AGGCGGTGCTTGTTCCTCAG & GGCTACAGGCTTGTCACTCG \\
PPAR- $\gamma$ & TGAATGTCGTGTCTGTGGAG & CAGCGGACTCTGGATTCAGC \\
GAPDH & GTACGTCGTGGAGTCCACTG & ATGGACTGTGGTCATGAG
\end{tabular}

COX, cyclooxygenase; IL, interleukin; TNF, tumor necrosis factor; PPAR, peroxisome proliferator-activated receptor; GAPDH, glyceraldehyde-3-phosphate dehydrogenase. 
GC samples using a modified TRI reagent (Sigma Chemical, St. Louis, MO, USA) according to the manufacturer's instructions. RNA concentration and purity were measured using a NanoDrop Spectrophotometer (NO-100; Biolab, Belmont, Australia). One microgram of total RNA was reverse-transcribed to cDNA using ImProm-II reverse transcriptase (Promega, Madison, WI, USA), and the CDNA was subjected to polymerase chain reaction amplification by specific primers (Table 1). Quantitative real-time polymerase chain reaction was carried out using SsoFast EvaGreen Supermix (Bio-Rad, Hercules, CA, USA), consisting of an initial denaturation step of $94^{\circ} \mathrm{C}$ for 30 seconds, followed by 30 amplification cycles at $94^{\circ} \mathrm{C}$ for 5 seconds, $50^{\circ} \mathrm{C}$ for 10 seconds, and $72^{\circ} \mathrm{C}$ for 10 seconds. Quantification of gene expression was performed only in the linear range of each primer pair. The delta CT method was used to quantify changes in the expression of each specific gene normalized to the expression of the housekeeping gene GAPDH [19].

\section{Statistical analysis}

Results were reported as the mean \pm standard deviation. The MannWhitney test was used to compare the mean values. Correlations between expressions of the various parameters were tested using the nonparametric Spearman correlation. Statistical significance was defined as $p<0.05$. SPSS for Windows ver. 11.0 (SPSS Inc., Chicago, IL, USA) was used for all analyses.

\section{Results}

The demographics and clinical outcomes of the PCOS and control groups are summarized in Table 2. The PCOS and control groups did

Table 2. Basal characteristics and reproductive outcomes

\begin{tabular}{lccc}
\hline Variable & PCOS $(\mathrm{n}=9)$ & Control $(\mathrm{n}=6)$ & $p$-value \\
\hline Age $(\mathrm{yr})$ & $31.4 \pm 1.1$ & $33.0 \pm 0.8$ & 0.066 \\
$\mathrm{BMI}\left(\mathrm{kg} / \mathrm{m}^{2}\right)$ & $24.6 \pm 1.3$ & $26.0 \pm 3.1$ & 0.950 \\
$\mathrm{FSH}(\mathrm{IU} / \mathrm{L})$ & $7.0 \pm 0.6$ & $7.6 \pm 0.4$ & 0.485 \\
$\mathrm{LH} / \mathrm{FSH}$ & $1.3 \pm 0.3$ & $0.7 \pm 0.2$ & 0.041 \\
AMH $(\mathrm{ng} / \mathrm{mL})$ & $11.17 \pm 1.87$ & $8.76 \pm 2.69$ & 0.145 \\
Total hMG $(\mathrm{IU})$ & $2,328.1 \pm 299.2$ & $2,612.5 \pm 196.9$ & 0.388 \\
Oocytes collected & $20.0 \pm 3.6$ & $16.3 \pm 3.4$ & 0.607 \\
Fertilization rate $(\%)$ & $67.6 \pm 6.9$ & $69.9 \pm 8.4$ & 0.776 \\
Good embryos & $8.2 \pm 6.1$ & $7.7 \pm 4.4$ & 0.848 \\
Embryos transferred & $2.4 \pm 0.2$ & $2.5 \pm 0.2$ & 0.864 \\
Clinical PR/cycle (\%) & 71.4 & 80.0 & 0.604 \\
Ongoing PR/cycle (\%) & 42.9 & 80.0 & 0.287 \\
\hline
\end{tabular}

Values are presented as mean \pm standard deviation unless otherwise indicated.

PCOS, polycystic ovary syndrome; BMI, body mass index; FSH, follicle-stimulating hormone; LH, luteinizing hormone; $\mathrm{AMH}$, anti-Müllerian hormone; hMG, human menopausal gonadotropin; PR, pregnancy rate. not significantly differ in age, body mass index, or the baseline hormonal profiles except for the ratio of luteinizing hormone to folliclestimulating hormone, which was significantly higher in the PCOS group. All patients underwent ICSI. There were also no significant differences in the ovarian stimulation profiles, including the total gonadotropin dose, the number of retrieved oocytes, the number of embryo transferred, and the number of good embryos. The clinical pregnancy rate and ongoing pregnancy rate were lower in the PCOS group, but these differences were not statistically significant.

The mRNA expression of PPAR- $\gamma$, COX-2, IL-6, and TNF- $\alpha$ in GCs from stimulated follicles in the PCOS and control groups is shown in Figure 1. PPAR- $\gamma$ and COX-2 mRNA was significantly downregulated in the GCs of PCOS women compared with controls $(p=0.034$ and $p=$ 0.018 , respectively), but IL-6 and TNF-a mRNA levels did not show significant differences.

The expression of PPAR- $\gamma$, COX-2, IL-6, and TNF- $\alpha$ in GCs was not correlated with clinical characteristics or oocyte developmental quality, as assessed by parameters including the number of oocytes, the number of transferred embryos, the fertilization rate, the rate of good embryos, and the clinical pregnancy rate (data not shown). Spearman correlation analysis indicated a significant positive association among the mRNA levels of PPAR- $\gamma$, COX-2, IL-6, and TNF- $a$ in GCs (Figure 2).
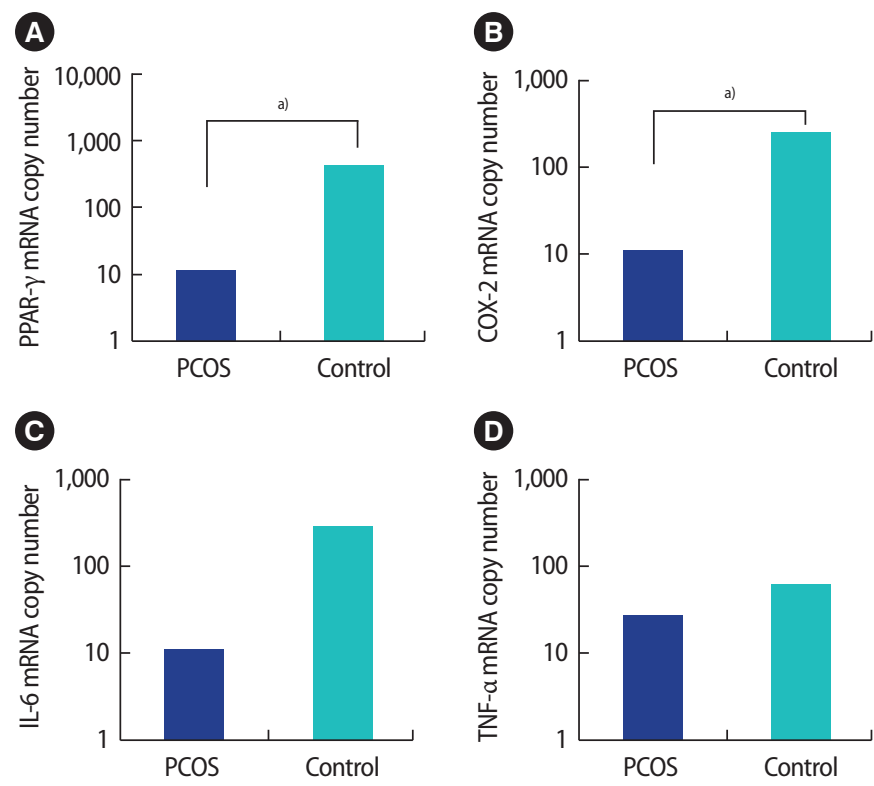

Figure 1. The mRNA expression of (A) PPAR- $\gamma$, (B) COX-2, (C) IL-6, and (D) TNF-a in granulosa cells from stimulated follicles in polycystic ovary syndrome (PCOS) and non-PCOS women. The mRNA copy number is given to allow comparison. PPAR- $\gamma$ and COX-2 mRNA was significantly downregulated in the granulosa cells of PCOS women compared with controls ( $p=0.034$ and $p=0.018$, respectively), but IL-6 and TNF-a mRNA did not show significant differences. PPAR, peroxisome proliferator-activated receptor; COX, cyclooxygenase; IL, interleukin; TNF, tumor necrosis factor. ${ }^{a)} p<0.01$. 

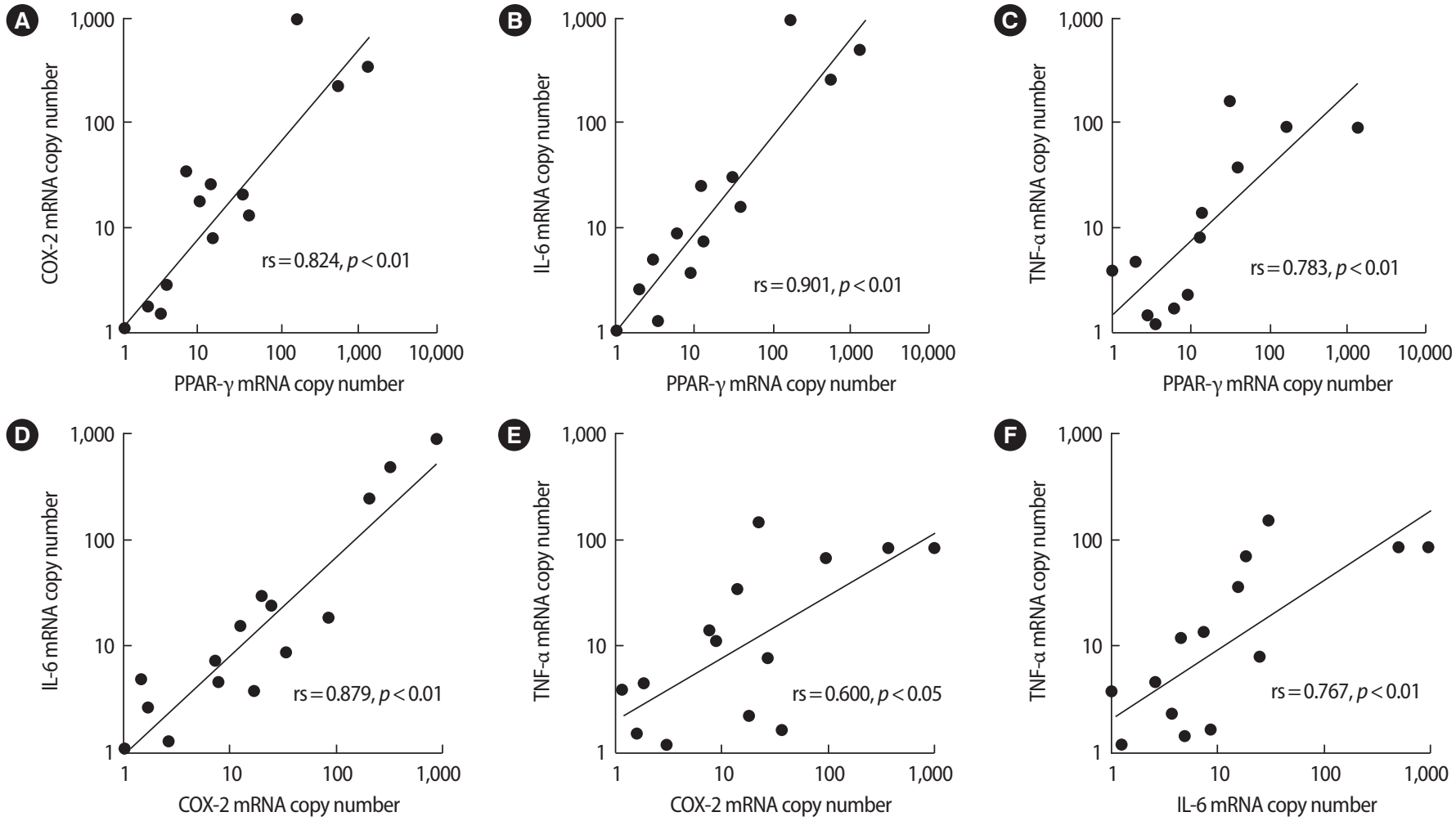

Figure 2. Spearman rank correlation (rs) between the mRNA expression levels of (A) PPAR- $\gamma$ and COX-2, (B) PPAR- $\gamma$ and IL-6, (C) PPAR- $\gamma$ and TNF-a, (D) COX-2 and IL-6, (E) COX-2 and TNF- $a$, and (F) IL-6 and TNF- $\alpha$ in granulosa cells. Spearman correlation analysis indicated a significant positive association among the levels of PPAR- $y$, COX-2, IL-6, and TNF- $a$ mRNA in granulosa cells. COX, cyclooxygenase; PPAR, peroxisome proliferator-activated receptor; IL, interleukin; TNF, tumor necrosis factor.

\section{Discussion}

In order to explore the dysregulation of GCs in PCOS, we investigated the mRNA expression patterns of PPAR- - , COX-2, and proinflammatory cytokines in the GCs of PCOS patients undergoing controlled ovarian stimulation. Although little is known about the factors that control the progression of follicle growth, it is clear that local factors are important and that GCs are primarily responsible for the regulation of FF content. We focused on genes responsive to PPAR- $\gamma$ signaling, which is critical for normal ovarian function [9]. When compared to normal ovaries, the majority of the differentially expressed genes were downregulated in PCOS ovaries, suggesting that several processes that are activated by these genes are in an off-state [20]. Moreover, considering the treatment effect of a synthetic agonist of PPAR- $\gamma$ [10], lower levels of PPAR- $\gamma$ expression are anticipated in PCOS. However, controversies exist about the expression level of PPAR- $\gamma$ in PCOS. Some studies have reported upregulation of PPAR- $\gamma$ in ovary and cumulus cells [20,21]; some have reported no significant differences in GCs [22]; and some reported downregulation in the GCs of women with PCOS [11]. We found that PPAR-y expression was downregulated in the GCs of women with PCOS. The reason for the inconsistent results regarding PPAR- $\gamma$ expression and PCOS is unclear, but it might be due to small sample sizes and the heterogeneity of PCOS.

An association between PPAR- $\gamma$ and COX-2 has been reported [23], but few studies on COX-2 expression in PCOS have been published. Sander et al. [24] showed that the levels of COX-2 mRNA did not change in PCOS follicles as compared to controls. Arif et al. [25] reported that COX-2 was upregulated twofold in polycystic rat ovaries in comparison to superovulated rat ovaries during ovulation. In contrast, our results showed that COX-2 mRNA was downregulated in the GCs of women with PCOS.

Imbalances between proinflammatory and anti-inflammatory cytokines closely linked to insulin resistance may be involved in the pathogenesis of PCOS [26]. TNF- $a$ and IL-6 are important proinflammatory cytokines that may influence ovarian function [27]. Infertile women with PCOS were found to have higher serum and FF concentrations of TNF- $\alpha$ and IL- 6 than controls $[9,12,28,29]$. PPAR- $\gamma$ was demonstrated to control a unique network of downstream genes, including those encoding for IL-6 and TNF- $a$ [30]. There is a presumption that chronic low-grade inflammation, represented by TNF- $a$ and IL-6, may have a harmful effect on the outcome of in vitro fertilization. 
However, our results showed that IL-6 and TNF-a mRNA expression in GCs did not show significant differences between the PCOS and control groups, and there was no significant correlation between the expression of these genes and indicators of oocyte developmental quality, including the fertilization rate and the rate of good embryos. Additionally, the expression level of PPAR- $\gamma$ and chronic low-grade inflammation markers did not show a negative correlation (Figure 2).

Despite the increasing clinical use of PPAR- $\gamma$ agonists to treat PCOS, the mechanisms by which PPAR- $\gamma$ agonists exert their effects remain unclear. PPAR- $\gamma$ agonist administration may influence ovarian function via numerous diverse mechanisms that may or may not be directly related to insulin and insulin-like growth factor signaling. Rosiglitazone was recently shown to significantly increase PPAR- $\gamma$ mRNA and to decrease TNF-a in human granulosa-lutein cells in vitro, as well as to decrease IL-6 secretion, although not significantly [31].

This study has some limitations. A systematic evaluation of metabolic abnormalities was not performed, meaning that other confounding factors may have been present. Although no significant difference in body weight was found between the study and control groups, a body weight-matched study was not performed. This study included a relatively small number of cases, and the PCOS study group did not contain multiple PCOS phenotypes. An important limitation is that we did not also perform further experiments using in vitro-cultured GCs to validate whether dysregulation of PPAR- $\gamma$ and COX-2 is intrinsic.

Based on the knowledge that GC dysregulation may affect the ovarian follicular environment, our results showed alterations of the follicular environment in PCOS. The present data may contribute to a better understanding of dysregulated pathways in PCOS. Further studies with a larger sample size and with administration of a PPAR- $\psi$ agonist both in vivo and in vitro are necessary to conclusively assess the potential role of PPAR- $\gamma$ and proinflammatory factors in the follicular environment of $\mathrm{PCOS}$.

\section{Conflict of interest}

No potential conflict of interest relevant to this article was reported.

\section{Acknowledgments}

The authors are grateful to Jung Hyun Kim for his impressive support with laboratory procedures.

\section{References}

1. Ehrmann DA. Polycystic ovary syndrome. N Engl J Med 2005;352: 1223-36.
2. Legro RS, Barnhart HX, SchlaffWD, Carr BR, Diamond MP, Carson $\mathrm{SA}$, et al. Clomiphene, metformin, or both for infertility in the polycystic ovary syndrome. N Engl J Med 2007;356:551-66.

3. Franks S, Stark J, Hardy K. Follicle dynamics and anovulation in polycystic ovary syndrome. Hum Reprod Update 2008;14:36778.

4. Swain JE, Pool TB. ART failure: oocyte contributions to unsuccessful fertilization. Hum Reprod Update 2008;14:431-46.

5. Kenigsberg S, Bentov Y, Chalifa-Caspi V, Potashnik G, Ofir R, Birk OS. Gene expression microarray profiles of cumulus cells in lean and overweight-obese polycystic ovary syndrome patients. Mol Hum Reprod 2009;15:89-103.

6. Kang S, Tsai LT, Rosen ED. Nuclear mechanisms of insulin resistance. Trends Cell Biol 2016;26:341-51.

7. Froment P, Gizard F, Defever D, Staels B, Dupont J, Monget P. Peroxisome proliferator-activated receptors in reproductive tissues: from gametogenesis to parturition. J Endocrinol 2006;189:199209.

8. Huang Z, Zhou X, Nicholson AC, Gotto AM Jr, Hajjar DP, Han J. Activation of peroxisome proliferator-activated receptor-alpha in mice induces expression of the hepatic low-density lipoprotein receptor. Br J Pharmacol 2008;155:596-605.

9. Komar CM. Peroxisome proliferator-activated receptors (PPARs) and ovarian function: implications for regulating steroidogenesis, differentiation, and tissue remodeling. Reprod Biol Endocrinol 2005;3:41.

10. Yki-Jarvinen H. Thiazolidinediones. N Engl J Med 2004;351:110618.

11. Qu F, Wang FF, Yin R, Ding GL, El-Prince M, Gao Q, et al. A molecular mechanism underlying ovarian dysfunction of polycystic ovary syndrome: hyperandrogenism induces epigenetic alterations in the granulosa cells. J Mol Med (Berl) 2012;90:911-23.

12. Minge CE, Ryan NK, Van Der Hoek KH, Robker RL, Norman RJ. Troglitazone regulates peroxisome proliferator-activated receptors and inducible nitric oxide synthase in murine ovarian macrophages. Biol Reprod 2006;74:153-60.

13. Vural P, Degirmencioglu S, Saral NY, Akgul C. Tumor necrosis factor alpha (-308), interleukin-6 (-174) and interleukin-10 (-1082) gene polymorphisms in polycystic ovary syndrome. Eur J Obstet Gynecol Reprod Biol 2010;150:61-5.

14. Amalfi S, Velez LM, Heber MF, Vighi S, Ferreira SR, Orozco AV, et al. Prenatal hyperandrogenization induces metabolic and endocrine alterations which depend on the levels of testosterone exposure. PLoS One 2012;7:e37658.

15. Subbaramaiah K, Lin DT, Hart JC, Dannenberg AJ. Peroxisome proliferator-activated receptor gamma ligands suppress the transcriptional activation of cyclooxygenase-2: evidence for in- 
volvement of activator protein-1 and CREB-binding protein/ p300. J Biol Chem 2001;276:12440-8.

16. Lim H, Paria BC, Das SK, Dinchuk JE, Langenbach R, Trzaskos JM, et al. Multiple female reproductive failures in cyclooxygenase 2-deficient mice. Cell 1997;91:197-208.

17. Rotterdam ESHRE/ASRM-Sponsored PCOS Consensus Workshop Group. Revised 2003 consensus on diagnostic criteria and longterm health risks related to polycystic ovary syndrome. Fertil Steril 2004;81:19-25.

18. Kim HJ, Kim CH, Lee SM, Choe SA, Lee JY, Jee BC, et al. Outcomes of preimplantation genetic diagnosis using either zona drilling with acidified Tyrode's solution or partial zona dissection. Clin Exp Reprod Med 2012;39:118-24.

19. Livak KJ, Schmittgen TD. Analysis of relative gene expression data using real-time quantitative PCR and the 2(-delta delta C(T)) method. Methods 2001;25:402-8.

20. Jansen E, Laven JS, Dommerholt HB, Polman J, van Rijt C, van den Hurk $C$, et al. Abnormal gene expression profiles in human ovaries from polycystic ovary syndrome patients. Mol Endocrinol 2004;18:3050-63.

21. Haouzi D, Assou S, Monzo C, Vincens C, Dechaud H, Hamamah S. Altered gene expression profile in cumulus cells of mature MII oocytes from patients with polycystic ovary syndrome. Hum Reprod 2012;27:3523-30.

22. Zaree M, Shahnazi V, Fayezi S, Darabi M, Mehrzad-Sadaghiani M, Darabi M, et al. Expression levels of PPARy and CYP-19 in polycystic ovarian syndrome primary granulosa cells: influence of $\omega$-3 fatty acid. Int J Fertil Steril 2015;9:197-204.

23. Ramer R, Heinemann K, Merkord J, Rohde H, Salamon A, Linnebacher $M$, et al. COX-2 and PPAR- $\gamma$ confer cannabidiol-induced apoptosis of human lung cancer cells. Mol Cancer Ther 2013;
12:69-82.

24. Sander VA, Hapon MB, Sicaro L, Lombardi EP, Jahn GA, Motta AB. Alterations of folliculogenesis in women with polycystic ovary syndrome. J Steroid Biochem Mol Biol 2011;124:58-64.

25. Arif M, Thakur SC, Datta K. Disrupted hyaluronan binding protein 1 (HABP1) expression: one of the key mediator for ovarian dysfunction in polycystic ovary rat. Mol Cell Biochem 2015;398:23344.

26. Elkind-Hirsch KE. Thiazolidinediones for the therapeutic management of polycystic ovary syndrome: impact on metabolic and reproductive abnormalities. Treat Endocrinol 2006;5:171-87.

27. Kim CH, Ahn JW, You RM, Kim SH, Chae HD, Kang BM. Pioglitazone treatment decreases follicular fluid levels of tumor necrosis factor- $a$ and interleukin- 6 in patients with polycystic ovary syndrome. Clin Exp Reprod Med 2011;38:98-102.

28. Amato G, Conte M, Mazziotti G, Lalli E, Vitolo G, Tucker AT, et al. Serum and follicular fluid cytokines in polycystic ovary syndrome during stimulated cycles. Obstet Gynecol 2003;101:117782.

29. Samy N, Hashim M, Sayed M, Said M. Clinical significance of inflammatory markers in polycystic ovary syndrome: their relationship to insulin resistance and body mass index. Dis Markers 2009;26:163-70.

30. Chinetti G, Fruchart JC, Staels B. Peroxisome proliferator-activated receptors (PPARs): nuclear receptors at the crossroads between lipid metabolism and inflammation. Inflamm Res 2000; 49:497-505.

31. Chen Q, Sun X, Chen J, Cheng L, Wang J, Wang Y, et al. Direct rosiglitazone action on steroidogenesis and proinflammatory factor production in human granulosa-lutein cells. Reprod Biol Endocrinol 2009;7:147. 